\title{
Tunneling Splittings in Water Clusters from Path Integral Molecular Dynamics
}

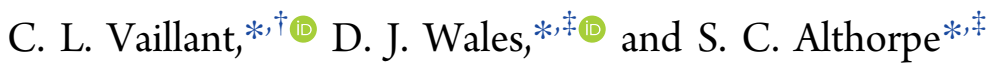 \\ ${ }^{\dagger}$ Laboratory of Theoretical Physical Chemistry, Institut des Sciences et Ingénierie Chimiques, École Polytechnique Fédérale de \\ Lausanne (EPFL), CH-1015 Lausanne, Switzerland \\ ${ }^{\ddagger}$ Department of Chemistry, University of Cambridge, Lensfield Road, Cambridge CB2 1EW, United Kingdom
}

Supporting Information

\begin{abstract}
We present calculations of tunneling splittings in selected small water clusters, based on a recently developed path integral molecular dynamics (PIMD) method. The groundrotational-state tunneling motions associated with the largest splittings in the water dimer, trimer, and hexamer are considered, and we show that the PIMD predictions are in very good agreement with benchmark quantum and experimental results. As the tunneling spectra are highly sensitive to both the details of the quantum dynamics and the potential energy surface, our calculations are a validation of the MB-Pol surface as well as the accuracy of PIMD. The favorable scaling of PIMD with system size paves the way for calculations of tunneling splittings in large, nonrigid molecular systems with motions that cannot be treated accurately by other methods, such as the semiclassical instanton.
\end{abstract}

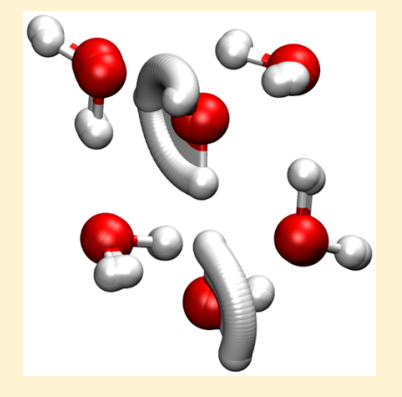

$\mathrm{T}$ he dynamics of water clusters have been the subject of intensive research efforts over the past few decades, due in part to the fundamental importance of water and the presence of water clusters in a number of different environments. ${ }^{1}$ The experimental study of cluster dynamics is particularly advanced, with the creation of far-infrared and terahertz lasers enabling detailed high-resolution spectroscopy studies of vibration-rotation-tunneling spectra for a range of cluster sizes. ${ }^{2}$ To interpret these experimental results quantitatively, however, requires sophisticated calculations of the dynamics to capture quantum effects such as tunneling, although assignments of spectra can be achieved from analysis of the permutation-inversion operations corresponding to fundamental rearrangement pathways. $^{3-8}$

Theoretical efforts in understanding the quantum dynamics of water clusters are particularly hampered by the unfavorable scaling of computational times as a function of system size. To date, only the water dimer has been treated variationally in full dimensionality, ${ }^{9}$ with major efforts in the past involving adiabatic separations of motions to facilitate calculations. ${ }^{10,11}$ Outside of variational methods, more approximate treatments exist, which can often predict tunneling splittings with useful accuracy. For example, early diffusion Monte Carlo calculations were able to obtain splittings for the water dimer and trimer. ${ }^{12}$ DMC remains the leading method for calculations of ground-state tunneling splittings, although a careful treatment of the fixed-node approximation is needed. ${ }^{13}$

Recent efforts involving path integral methods have become promising new avenues for accurately calculating tunneling splittings in ground rotational states, with instanton calculations (first introduced in theoretical chemistry to calculate reaction rates ${ }^{14}$ ) being particularly straightforward. ${ }^{15-19}$ Methods also exist for automating the instanton search ${ }^{20}$ and substantially improving the computation time required. ${ }^{21,22}$ However, due to the semiclassical approximation that is the hallmark of the instanton method, predictions of tunneling splittings can be inaccurate due to anharmonicities perpendicular to the instanton path and a poor description of low-barrier tunneling. ${ }^{23}$ For this reason, a new path integral molecular dynamics (PIMD) method was introduced, ${ }^{24}$ capable of obtaining higher-accuracy tunneling splittings for very nonrigid ("floppy") molecules and clusters. The method was extended to multiwell tunneling ${ }^{25}$ and later applied to the water dimer. ${ }^{26}$

In this contribution, we present PIMD calculations for a range of ground-state tunneling motions in water clusters, focusing on the motions that give rise to the largest splittings for each cluster. We show that the PIMD method can accurately reproduce the tunneling splittings of these motions, in contrast to the instanton predictions, which exhibit large discrepancies with reference values. This very good agreement over a wide range of cluster sizes would not be possible without accurate quantum dynamics predictions in combination with a high-quality potential energy surface (PES), such as the MB-Pol function (which truncates the many-body expansion at the 3 -body term), ${ }^{27,28}$ used throughout the present calculations.

We first summarize the theory required for the calculation of tunneling splittings using PIMD. The expressions presented here have previously been derived in detail elsewhere. ${ }^{24-26}$ Consider initially a double-well system, where the minimum $\mathbf{r}$ of a well is connected to the minimum $\mathbf{r}^{\prime}$ of another well by a symmetry operation $\hat{P}$, such that $\mathbf{r}^{\prime}=\hat{P} \mathbf{r}$. To find the tunneling

Received: October 7, 2019

Accepted: November 4, 2019

Published: November 4, 2019 
splitting, we write the ratio of two thermodynamic density matrices in the low temperature limit $\beta \rightarrow \infty$ (which allows us to neglect energy levels above the first-excited state)

$$
I(\beta)=\frac{\left\langle\mathbf{r}\left|\mathrm{e}^{-\beta H}\right| \hat{P} \boldsymbol{r}\right\rangle}{\left\langle\mathbf{r}\left|\mathrm{e}^{-\beta H}\right| \boldsymbol{r}\right\rangle} \approx \tanh \left[\frac{\Delta}{2}(\beta-\bar{\beta})\right]
$$

where $\bar{\beta}$ is known as the tunneling time, ${ }^{29} \Delta$ is the tunneling splitting, and $\mathrm{e}^{-\beta H}$ is the thermodynamic density operator for the Hamiltonian of the $f$-dimensional system

$$
\hat{H}=\sum_{j=1}^{f} \frac{\hat{p}_{j}^{2}}{2 m_{j}}+\hat{V}
$$

$I(\beta)$ can be accurately calculated using path integral methods, and hence the tunneling splitting can be obtained from eq 1 . As there are two free parameters, $\Delta$ and $\bar{\beta}$, it is necessary to calculate values of $I(\beta)$ for two values of $\beta$ and solve for the required tunneling splitting.

For more than two wells, eq 1 must be generalized to take into account the multiple tunneling paths. While it is possible to account for these multiple tunneling paths exactly for given values of $\beta,{ }^{25,26}$ it is much more convenient to be able to converge the PIMD calculations for each tunneling path separately. We therefore employ the concept of the tunneling matrix, W, first defined in ref 16 for instanton calculations of tunneling splittings, which can be derived from the molecular symmetry group assuming that the paths are completely independent (a good approximation at low temperature). ${ }^{30}$ Subsequently, it is possible to define a tunneling matrix element connecting wells $i$ and $j$ in terms of $I(\beta)$ in eq 1 such that $^{30}$

$$
h_{i j}=\lim _{\beta \rightarrow \infty} \frac{1}{\bar{\beta}-\beta} \tanh ^{-1}\left[I_{i j}(\beta)\right]
$$

In other words, we can calculate a full tunneling spectrum by considering a series of effective double-well calculations and diagonalizing the resulting tunneling matrix.

To calculate the density matrices in eq 1 with path integral techniques, it is convenient to first discretize the path into a "linear polymer" representation, where $N$ copies of the system (which we call "beads") sample the PES with an effective interbead coupling term representing the kinetic energy operator. Under this standard approximation using the Trotter-Suzuki theorem, ${ }^{31}$ the thermodynamic density matrices in the position representation between beads a and b can be written as ${ }^{29}$

$$
\rho(\mathbf{a}, \mathbf{b}, \beta) \approx\left(\prod_{j=1}^{f} \frac{m_{j}}{2 \pi \hbar^{2} \beta_{N}}\right)^{(N+1) / 2} \times \int \mathrm{d} \mathbf{r}_{1} \ldots \int \mathrm{d} \mathbf{r}_{N} \mathrm{e}^{-\beta_{N} U_{N}}
$$

where

$$
\begin{aligned}
U_{N}= & \sum_{i=1}^{N}\left[\sum_{j=1}^{f} \frac{1}{2} m_{j} \omega_{N}^{2}\left(r_{i, j}-r_{i+1, j}\right)^{2}+V\left(r_{i}\right)\right] \\
& +\sum_{j=1}^{f} \frac{1}{2} m_{j} \omega_{N}^{2}\left[\left(a_{j}-r_{1, j}\right)^{2}+\left(r_{N, j}-b_{j}\right)^{2}\right]
\end{aligned}
$$

Here, $f$ is the number of degrees of freedom (the number of atoms multiplied by the number of dimensions), $m_{j}$ is the mass of the atom for the jth degree of freedom, and $\omega_{N}=\left(\beta_{N} \hbar\right)^{-1}$. We have also set $V(\mathbf{a})=V(\mathbf{b})=0$ explicitly.

To evaluate the ratio of integrals in eq 4 efficiently, we use a thermostated molecular dynamics simulation combined with a thermodynamic integration. ${ }^{26}$ To use molecular dynamics simulations, we must first express eq 4 as an effective classical Hamiltonian system by inserting the identity, expressed as an integral over a Gaussian, into (4) $N \times f$ times to obtain

$$
\begin{aligned}
\rho(\vec{a}, \vec{b}, \beta) \approx & {\left[\prod_{j=1}^{f} \frac{2 \pi m_{j}}{\beta_{N}}\left(\prod_{i=1}^{N} \frac{m_{j}}{4 \pi^{2} \hbar^{2} \mu_{i j}}\right)\right]^{1 / 2} } \\
& \times \int \mathrm{d} \vec{p}_{1} \ldots \int \mathrm{d} \vec{p}_{N} \int \mathrm{d} \vec{r}_{1} \ldots \int \mathrm{d} \vec{r}_{N} \mathrm{e}^{-\beta_{N} H_{N}}
\end{aligned}
$$

where

$$
H_{N}=\sum_{i=1}^{N} \sum_{j=1}^{f} \frac{p_{i, j}^{2}}{2 \mu_{i j}}+U_{N}
$$

is the effective classical Hamiltonian with the ring polymer potential $U_{N}$ given in eq 5 and $p_{i, j}$ is the fictitious classical momentum for the $i$ th bead and $j$ th degree of freedom. The "bead mass", $\mu_{i j}$, is a free parameter and can be optimized to improve the sampling. ${ }^{26,32}$ We make the choice

$$
\mu_{j k}=\frac{m_{j} \omega_{k}^{2}}{\hbar^{2} \beta_{N}^{2}}
$$

where $\omega_{k}$ denotes the polymer mode frequency. ${ }^{24}$

Thermodynamic integration is achieved using a free energy function

$$
F\left(\lambda, \beta_{N}\right)=-\frac{1}{\beta_{N}} \ln \rho(\mathbf{a}, \mathbf{b}(\lambda), \beta)
$$

where $\lambda$ is a reaction coordinate $(\lambda=0,1$ for $\mathbf{r}=\mathbf{a}, \mathbf{b}$, respectively). From this definition, we can write

$$
\begin{aligned}
I(\beta) & =\exp \left[-\beta_{N}\left(F\left(\lambda=1, \beta_{N}\right)-F\left(\lambda=0, \beta_{N}\right)\right)\right] \\
& =\exp \left[-\beta_{N} \Delta F\right]
\end{aligned}
$$

where the free energy difference $\Delta F$ is now the quantity to be calculated. Following the well-known thermodynamic integration technique, we write

$$
\begin{aligned}
\Delta F & =\left.\int_{0}^{1} \mathrm{~d} \lambda^{\prime} \frac{\partial F}{\partial \lambda}\right|_{\lambda^{\prime}} \\
& =\int_{0}^{1} \mathrm{~d} \lambda^{\prime}\left\langle\frac{\partial H}{\partial \lambda}\right\rangle_{\lambda^{\prime}} \\
& =-\sum_{i=1}^{f} m_{i} \omega_{N}^{2} \int_{0}^{1} \mathrm{~d} \lambda^{\prime}\left\langle r_{i N}\right\rangle_{\lambda^{\prime}} \frac{\partial b_{i}}{\partial \lambda}
\end{aligned}
$$

where $\langle\cdots\rangle_{\lambda}$ denotes a thermodynamic ensemble average at a fixed value of $\lambda$. We perform the ensemble average by running a molecular dynamics (MD) simulation, with a Langevin thermostat coupled to the linear polymer for efficient sampling. ${ }^{26,33,34}$

In summary, the value of $I_{i j}(\beta)$ is calculated by varying the end bead of a linear polymer from well $i$ to well $j$ and performing a thermodynamic ensemble average of the estimator in (11) for each value of $\lambda$. In practice, it is 
convenient to take the reaction coordinate to follow the instanton path, ${ }^{15,16,26}$ and use Gauss-Legendre integration to evaluate the integral in (11). ${ }^{35}$ Using eq 3 , the relevant entries of the tunneling matrix can be obtained and the matrix diagonalized to give the required tunneling spectrum. The instanton pathways in some water clusters are well understood. $^{16,20,36,37}$ However, many water clusters have large splittings dominated by a single motion with small barriers, and the corresponding tunneling matrix elements are poorly reproduced by instanton calculations.

We first consider the water dimer, for which PIMD results already exist. ${ }^{26}$ However, the previous results suffered from a significant discrepancy in the largest motion, known as the "acceptor flip"; the instanton for this motion is shown in Figure 1a. The problem is due to the fact that rotations were not

(a)

(b)

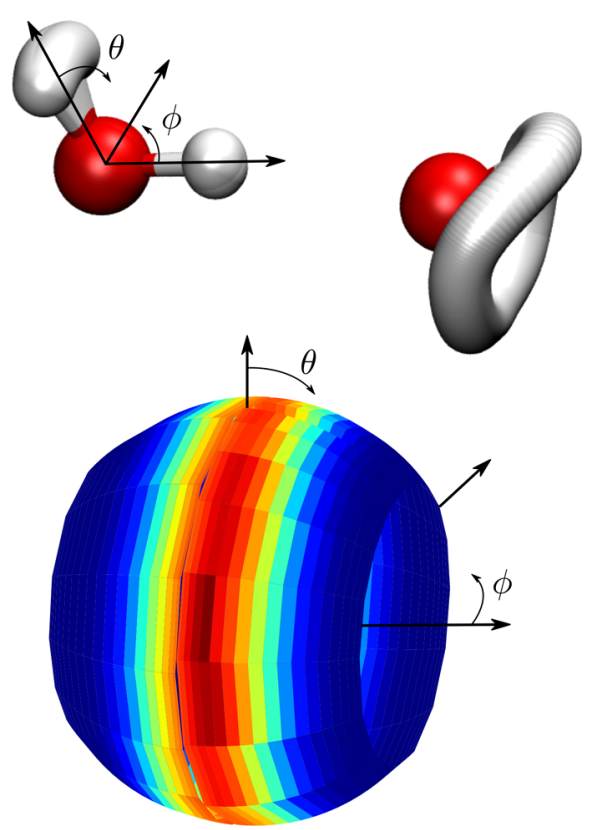

Figure 1. (a) Instanton pathway for the acceptor flip motion of the water dimer, with two Euler angles $\theta$ and $\phi$, corresponding to the rotation about the principal axis and an azimuthal rotation respectively, indicated. (b) Values of $I(\beta, \mathbf{\Omega})$ integrated over the final Euler angle $\eta$, as a function of $\theta$ and $\phi$. The peak occurs at a rotation of nearly $\theta=\pi / 2$, which is consistent with the overall rotation observed for an instanton with unfixed ends.

taken into account, and the acceptor flip couples very strongly to overall rotations of the water dimer about the principal axis. This strong coupling to rotations is not typically a problem in clusters, but in the dimer it leads to large errors. We note that no direct experimental measurement of this largest splitting in the dimer can be performed due to unfavorable selection rules.

When finding an instanton path, one can choose to fix the end beads in the relevant wells, such that the start and end structures are optimally aligned. However, the value of $I(\beta)$ for any given tunneling motion depends on the Euler angles $\boldsymbol{\Omega}=$ $(\theta, \phi, \eta) .^{30}$ This variation is shown in Figure $1 \mathrm{~b}$ for the acceptor flip (integrated over $\eta$ in order to project on a spherical surface), and a strong peak is observed near $\theta=\pi / 2$. In fact, this peak corresponds to the overall rotation that occurs when the end beads remain unfixed; in other words, the peak occurs at the path of minimum action, as expected.
In principle, to fully account for the rotational dependence of the tunneling, one would need to integrate over the Euler angles. ${ }^{30}$ However, it is difficult to sample efficiently enough to provide good statistics with PIMD. In the ground rotational state, we expect the rotational dependence to be less important. We therefore choose the instanton path obtained with unfixed ends and assume that this single point dominates the integral. For the larger clusters, we found that the choice of fixed or unfixed end beads did not significantly affect the results, although the convergence properties of the fixed-end paths were better.

We next turn to the water trimer, which is the smallest cluster without numerically exact variational results, although both theoretical ${ }^{4,12,16,38-42}$ and experimental ${ }^{43-45}$ values exist in the literature for comparison. We will focus specifically on the flip motion, where one of the dangling hydrogens flips orientation; the instanton pathway is shown in Figure 2. The

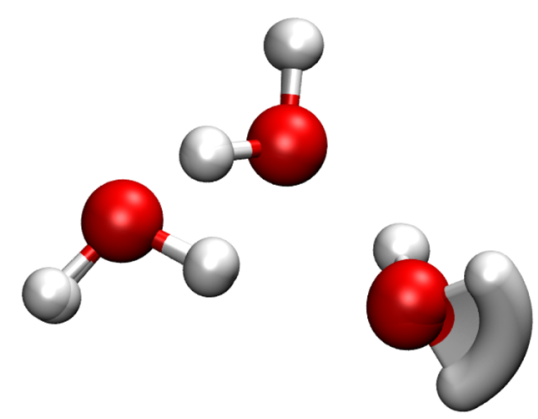

Figure 2. Instanton pathway for the flip rearrangement of the water trimer.

flip motion is particularly interesting because the zero-point energy actually lies above the barrier height, making the pathway quite anharmonic. As a result, the instanton prediction overestimates the tunneling matrix element by a factor of about $2 .{ }^{16}$ For the bifurcation tunnelling pathway ${ }^{4}$ the instanton method predicts the corresponding tunneling matrix elements much more accurately than for the flip (because the barriers are higher), so we do not consider this process here.

The full spectrum for the water trimer is relatively complicated: the flip produces a quartet splitting pattern, which is further split by other motions. Most analyses of the trimer consider the quartet splitting as a vibrational motion, with a corresponding quantum number $k$ ranging from 0 to 3 . We refer to the difference between the two outer branches as the "tunneling splitting", and the tunneling matrix element is a quarter of this value.

The last cluster we consider is the water hexamer in the prism configuration, where a recent study identified two concerted tunneling motions that give rise to experimentally observable splittings. ${ }^{36}$ The instanton calculations performed for this study gave an excellent prediction for the structure of the spectrum, namely, a doublet of triplets, and found that the rotational-tunneling coupling was negligible. However, the splittings were overestimated compared to the experimental values, and the source of this error was suggested as either the neglect of anharmonicity in the semiclassical approximation or inaccuracies in the PES.

We consider both concerted tunneling motions identified in the hexamer prism, referred to as the antigeared and geared motions, as shown in Figure 3a, b, respectively. The largest doublet splitting arises from the antigeared motion, while the 
(a)

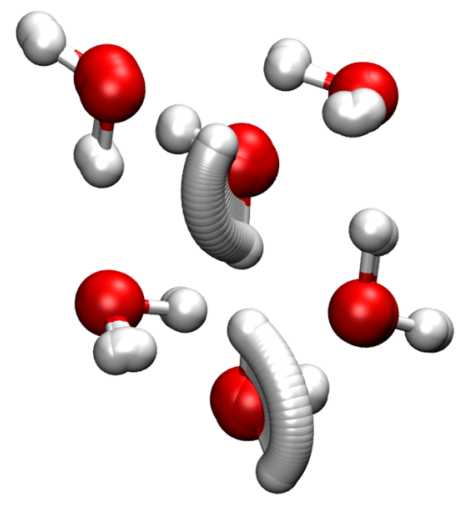

(b)

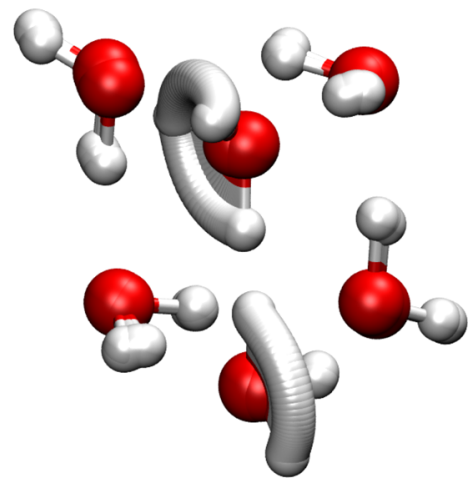

Figure 3. Instanton pathways for the (a) antigeared and (b) geared motions of the water hexamer prism.

geared pathway splits each branch of the doublet into a triplet. The instanton calculations overestimated both tunneling matrix elements by about a factor of 2 compared to the experimental values. ${ }^{36}$

The results of the PIMD calculations for the tunneling matrix elements associated with all the pathways considered above are shown in Table 1. Generally, the PIMD results agree

Table 1. Tunneling Matrix Elements Calculated with the PIMD and Instanton Methods ${ }^{a}$

\begin{tabular}{llcc}
\multicolumn{1}{c}{ pathway/cluster } & \multicolumn{1}{c}{ PIMD } & instanton & reference \\
dimer acceptor $\left(\mathrm{cm}^{-1}\right)$ & $3.0(1)$ & 5.5 & 3.0 \\
trimer flip $\left(\mathrm{cm}^{-1}\right)$ & $26(2)$ & 50 & 21.76 \\
hexamer geared $(\mathrm{MHz})$ & $0.12(1)$ & 0.11 & 0.073 \\
hexamer antigeared $(\mathrm{MHz})$ & $0.4(1)$ & 0.75 & 0.382
\end{tabular}

${ }^{a}$ The reference values of the tunneling matrix elements are variational results on the same PES for the water $\operatorname{dimer}^{27}$ and experimental results for the trimer ${ }^{45}$ and hexamer. ${ }^{36}$ The statistical uncertainty in the last significant figure for each quantity is shown in parentheses.

very well with the reference values for all the motions considered. The results for the water dimer, calculated with an instanton having unfixed ends, agree to within the statistical uncertainty with the variational calculations, showing that the integral over Euler angles is indeed dominated by the path of least action for the ground rotational state. Of course, this will not be the case for higher excited rotational states, and a more careful treatment of this angular integral (which is beyond the scope of this study) is needed.

The water hexamer results are particularly interesting, because the PIMD and instanton predictions for the geared pathway agree very well but disagree with experiment (the instanton result on the CC-pol surface produces a slightly worse disagreement). In contrast, the PIMD prediction for the antigeared tunneling matrix element is in excellent agreement with experiment but in disagreement with the instanton prediction. The combination of these results implies that the antigeared pathway may suffer from large anharmonic contributions neglected by the instanton. We note that both the hexamer values are slightly larger than the experimental values, which could be an artifact of poor convergence with the number of beads, but is more likely to come from uncertainties in the PES, possibly due to the truncation of the many-body expansion in MB-Pol.

In summary, we have demonstrated that path integral molecular dynamics gives very good predictions of tunneling splittings in a range of water clusters. For the water dimer, special care must be taken to ensure correct treatment of the coupling of overall rotations to the tunneling motion. A simple solution of unfixing the end beads for the instanton path was suggested, coinciding with the choice of Euler angles that minimize the quantum action. For the water trimer the flip was investigated, and the agreement with experiment for the tunneling matrix element is very good, although the values lie just outside the statistical uncertainty of the PIMD calculation. For the water hexamer, the tunneling matrix element for the geared pathway agrees well with the instanton predictions but disagrees with experiment, implying that anharmonicities are not an issue, but perhaps the PES requires four-body terms and higher for a quantitative agreement. The PIMD value for the antigeared tunneling matrix element, however, agrees well with experiment but disagrees with the instanton values, suggesting that contributions from anharmonic paths may be more important for this rearrangement. More generally, the PIMD method is complementary to the semiclassical instanton method and can be used when instanton predictions are inaccurate.

\section{ASSOCIATED CONTENT}

\section{S Supporting Information}

The Supporting Information is available free of charge on the ACS Publications website at DOI: 10.1021/acs.jpclett.9b02951.

Table of the parameters and results of the PIMD calculations used to calculate the splittings in Table 1, including a discussion of the convergence (PDF)

\section{AUTHOR INFORMATION}

\section{Corresponding Authors}

*C.L.V. E-mail: christophe.vaillant@epfl.ch.

*D.J.W. E-mail: dw34@cam.ac.uk.

*S.C.A. E-mail: sca10@cam.ac.uk.

ORCID $\odot$

C. L. Vaillant: 0000-0002-7528-8753

D. J. Wales: 0000-0002-3555-6645

\section{Notes}

The authors declare no competing financial interest.

\section{ACKNOWLEDGMENTS}

We thank Jeremy Richardson for helpful comments on the manuscript. This project was funded by the United Kingdom Engineering and Physical Sciences Research Council (EPSRC). 


\section{REFERENCES}

(1) Liu, K.; Cruzan, J. D.; Saykally, R. J. Water Clusters. Science 1996, 271, 929.

(2) Keutsch, F. N.; Saykally, R. J. Water Clusters: Untangling the Mysteries of the Liquid, One Molecule at a Time. Proc. Natl. Acad. Sci. U. S. A. 2001, 98, 10533.

(3) Wales, D. J. In Theory of Atomic and Molecular Clusters; Jellinek, J., Ed.; Springer-Verlag: Heidelberg, 1999; p 86.

(4) Wales, D. J. Theoretical Study of the Water Trimer. J. Am. Chem. Soc. 1993, 115, 11180.

(5) Wales, D. J.; Walsh, T. R. Theoretical Study of the Water Pentamer. J. Chem. Phys. 1996, 105, 6957-6971.

(6) Wales, D. J.; Walsh, T. R. Theoretical Study of the Water Tetramer. J. Chem. Phys. 1997, 106, 7193-7207.

(7) Wales, D. J. In Recent Theoretical and Experimental Advances in Hydrogen-Bonded Clusters; Xantheas, S., Ed.; Kluwer: Dordrecht, 1997.

(8) Wales, D. J. In Advances in Molecular Vibrations and Collision Dynamics; Bowman, J. M., Bačić, Z., Eds.; JAI Press: Stamford, 1998; Vol. 3; pp 365-396.

(9) Wang, X. G.; Carrington, T. Using Monomer Vibrational Wavefunctions to Compute Numerically Exact (12D) Rovibrational Levels of Water Dimer. J. Chem. Phys. 2018, 148, 074108.

(10) Groenenboom, G. C.; Wormer, P. E. S.; van der Avoird, A. Water Pair Potential of Near Spectroscopic Accuracy. II. VibrationRotation-Tunneling Levels of the Water Dimer. J. Chem. Phys. 2000, 113,6702 .

(11) Leforestier, C.; Gatti, F.; Fellers, R. S.; Saykally, R. J. Determination of a Flexible (12D) Water Dimer Potential Via Direct Inversion of Spectroscopic Data. J. Chem. Phys. 2002, 117, 8710.

(12) Gregory, J. K.; Clary, D. C. Calculations of the Tunneling Splittings in Water Dimer and Trimer Using Diffusion Monte Carlo. J. Chem. Phys. 1995, 102, 7817.

(13) McCoy, A. B.; Hinkle, C. E.; Petit, A. S. In Advances in Quantum Monte Carlo; Tanaka, S., Rothstein, S. M., Lester, W. A., Eds.; American Chemical Society Washington, DC, 2012; Chapter 12, pp 145-155.

(14) Miller, W. H. Semiclassical Limit of Quantum Mechanical Transition State Theory for Nonseparable Systems. J. Chem. Phys. 1975, 62, 1899.

(15) Richardson, J. O.; Althorpe, S. C. Ring-polymer Instanton Method for Calculating Tunneling Splittings. J. Chem. Phys. 2011, 134, 054109.

(16) Richardson, J. O.; Althorpe, S. C.; Wales, D. J. Instanton Calculations of Tunneling Splittings for Water Dimer and Trimer. J. Chem. Phys. 2011, 135, 124109.

(17) Mil'nikov, G. V.; Nakamura, H. Practical Implementation of the Instanton Theory for the Ground-State Tunneling Splitting. J. Chem. Phys. 2001, 115, 6881.

(18) Mil'nikov, G. V.; Nakamura, H. Tunneling Splitting and Decay of Metastable States in Polyatomic Molecules: Invariant Instanton Theory. Phys. Chem. Chem. Phys. 2008, 10, 1374.

(19) Siebrand, W.; Smedarchina, Z.; Zgierski, M. Z.; FernandezRamos, A. Proton Tunnelling in Polyatomic Molecules: A DirectDynamics Instanton Approach. Int. Rev. Phys. Chem. 1999, 18, 5.

(20) Vaillant, C. L.; Althorpe, S. C.; Wales, D. J. Path Integral Energy Landscapes for Water Clusters. J. Chem. Theory Comput. 2019, $15,33$.

(21) Cvitas, M. T.; Althorpe, S. C. Locating Instantons in Calculations of Tunneling Splittings: The Test Case of Malonaldehyde. J. Chem. Theory Comput. 2016, 12, 787.

(22) Cvitas, M. T. Quadratic String Method for Locating Instantons in Tunneling Splitting Calculations. J. Chem. Theory Comput. 2018, 14, 1487-1500.

(23) Richardson, J. O. Ring-Polymer Instanton Theory. Int. Rev. Phys. Chem. 2018, 37, 171.

(24) Mátyus, E.; Wales, D. J.; Althorpe, S. C. Quantum Tunneling Splittings from Path-Integral Molecular Dynamics. J. Chem. Phys. 2016, 144, 114108.
(25) Mátyus, E.; Althorpe, S. C. Calculating Splittings Between Energy Levels of Different Symmetry Using Path-Integral Methods. J. Chem. Phys. 2016, 144, 114109.

(26) Vaillant, C. L.; Wales, D. J.; Althorpe, S. C. TunnelingSplittings from Path-Integral Molecular Dynamics Using a Langevin Thermostat. J. Chem. Phys. 2018, 148, 234102.

(27) Babin, V.; Leforestier, C.; Paesani, F. Development of a "First Principles" Water Potential with Flexible Monomers: Dimer Potential Energy Surface, VRT Spectrum, and Second Virial Coefficient. J. Chem. Theory Comput. 2013, 9, 5395.

(28) Babin, V.; Medders, G. R.; Paesani, F. Development of a First Principles Water Potential with Flexible Monomers. II: Trimer Potential Energy Surface, Third Virial Coefficient, and Small Clusters. J. Chem. Theory Comput. 2014, 10, 1599.

(29) Ceperley, D. M. Path Integrals in the Theory of Condensed Helium. Rev. Mod. Phys. 1995, 67, 279.

(30) Vaillant, C. L.; Cvitaš, M. T. Rotation-Tunneling Spectrum of the Water Dimer from Instanton Theory. Phys. Chem. Chem. Phys. 2018, 20, 26809.

(31) Trotter, H. F. On the Product of Semi-Groups of Operators. Proc. Am. Math. Soc. 1959, 10, 545.

(32) Ceriotti, M.; Parrinello, M.; Markland, T. E.; Manolopoulos, D. E. Efficient Stochastic Thermostatting of Path Integral Molecular Dynamics. J. Chem. Phys. 2010, 133, 124104.

(33) Constable, S.; Schmidt, M.; Ing, C.; Zheng, T.; Roy, P.-N. Langevin Equation Path Integral Ground State. J. Phys. Chem. A 2013, $117,7461$.

(34) Schmidt, M.; Roy, P.-N. Path Integral Molecular Dynamic Simulation of Flexible Molecular Systems in Their Ground State: Application to the Water Dimer. J. Chem. Phys. 2018, 148, 124116.

(35) Press, W. H.; Teukolsky, S. A.; Vetterling, W. T.; Flannery, B. P. Numerical Recipies in Fortran; Cambridge University Press: Cambridge, UK, 1992.

(36) Richardson, J. O.; Pérez, C.; Lobsiger, S.; Reid, A. A.; Temelso, B.; Shields, G. C.; Kisiel, Z.; Wales, D. J.; Pate, B. H.; Althorpe, S. C. Concerted Hydrogen-Bond Breaking by Quantum Tunneling in the Water Hexamer Prism. Science 2016, 351, 1310.

(37) Richardson, J. O.; Wales, D. J.; Althorpe, S. C.; McLaughlin, R. P.; Viant, M. R.; Shih, O.; Saykally, R. J. Investigation of Terahertz Vibration-Rotation Tunneling Spectra for the Water Octamer. J. Phys. Chem. A 2013, 117, 6960.

(38) Olthof, E. H. T.; van der Avoird, A.; Wormer, P. E. S.; Liu, K.; Saykally, R. J. Tunneling Dynamics, Symmetry, and Far-Infrared Spectrum of the Rotating Water Trimer. II. Calculations and Experiments. J. Chem. Phys. 1996, 105, 8051.

(39) Sabo, D.; Bačić, Z.; Bürgi, T.; Leutwyler, S. 3-Dimensional Model Calculation of Torsional Levels of $(\mathrm{H} 2 \mathrm{O})(3)$ and (D2O)(3). Chem. Phys. Lett. 1995, 244, 283-294.

(40) Sabo, D.; Bačić, Z.; Bürgi, T.; Leutwyler, S. Three-Dimensional Model Treatment of the Torsional Levels of Isotopic Water Trimers. Chem. Phys. Lett. 1996, 261, 318-328.

(41) Sabo, D.; Bačić, Z.; Graf, S.; Leutwyler, S. Calculated and Experimental Rotational Constants of $(\mathrm{H} 2 \mathrm{O})(3)$ : Effects of Intermolecular Torsional and Symmetric Stretching Excitations. J. Chem. Phys. 1999, 111, 10727-10729.

(42) Takahashi, M.; Watanabe, Y.; Taketsugu, T.; Wales, D. J. An Ab Initio Study of Tunneling Splittings in the Water Trimer. J. Chem. Phys. 2005, 123, 044302.

(43) Pugliano, N.; Saykally, R. J. Measurement of Quantum Tunneling Between Chiral Isomers of the Cyclic Water Trimer. Science 1992, 257, 1937.

(44) Keutsch, F. N.; Saykally, R. J. Bifurcation Tunneling Dynamics in the Water Trimer. J. Chem. Phys. 2002, 117, 8823.

(45) Keutsch, F. N.; Cruzan, J. D.; Saykally, R. J. The Water Trimer. Chem. Rev. 2003, 103, 2533. 